\title{
SÍNDROME DE WEST EVOLUINDO PARA SINDROME DE LENNOX-GASTAUT EM PACIENTE COM CISTICERCOSE CEREBRAL
}

\author{
Mario Luiz Frochtengarten * \\ OzIR SCARANTE **
}

Embora o quadro clínico da hoje chamada síndrome de West tenha sido descrito em 1841, foi só a partir de 1952, quando o casal Gibbs e Gastaut descreveram o quadro eletrencefalográfico da hipsarritmia, que esta afecção passou a ser melhor estudada. Desde então foram publicados numerosos trabalhos a respeito, tendo sido descritas etiologias as mais variadas, como sejam malformações congênitas, traumatismos obstétricos, moléstias metabólicas, moléstias degenerativas, anóxia neonatal, hematomas, meningites e encefalites perinatais, síndromes neuro-cutâneas, mongolismo, toxoplasmose, vacinação tríplice e por administração de 5-hidroxitriptofano ${ }^{1,2,3,4,5,8,9}$.

Quanto à síndrome hoje conhecida como de Lennox-Gastaut, o complexo ponta-onda lenta já havia sido descrito em 1939, com o nome de Variante do Pequeno Mal, mas somente a partir de 1966, após o Colóquio Europeu de Informação Eletrencefalográfica, realizado em Marselha, é que esta entidade vem sendo melhor estudada. São citadas como etiologias da síndrome de Lennox-Gastaut, as malformações congênitas, os traumatismos de parto, hemorragias cerebrais neonatais, kernicterus, distrofias alimentares, toxoplasmose, síndromes neuro-cutâneas, tumores cerebrais, meningo-encefalites e, também, casos evoluídos a partir de síndromes de West primitivas $6,7,10,11,12$.

Registramos aqui o caso de uma criança na qual se desenvolveu um quadro característico de sindrome de West que, ulteriormente, evoluiu para uma sindrome de Lennox-Gastaut; o valor do registro se prende à etiologia inusitada - neurocisticercose - que se apurou no caso.

OB S E R V A A O

O.D.L., sexo feminino, branca, com 6 anos de idade. A paciente nascida de gravidez e parto normais, com peso de $2.900 \mathrm{~g}$, sem dados que sugiram anóxia perinatal. Sustentou a cabeça com 5 meses; emitiu as primeiras palavras e andou sem apoio aos 17 meses. A avó da criança apresentava tenlase e tinha contacto constante com a paciente. Em 14-5-68, com 18 meses de idade, durante processo

* Eletrencefalografista do Hospital dos Servidores Públicos do Estado de São Paulo (Prof. Roberto Melaragno Filho).

** Chefe da Seção de Diagnóstico e Terapêutica da Clínica Neurológica do Hospital dos Servidores Públicos do Estado de São Paulo (Prof. Roberto Melaragno Filho). 
infeccioso de vias aéreas superiores, a paciente apresentou desidratação e hipertermia, ocorrendo nesta ocasiāo uma crise convulsiva dimidiada à direita, em sono, com duração de uma hora. Um mês após veio á consulta pela primeira vez. Ao exame, mostrava-se bem nutrida, hidratada, contactuando bem com o meio ambiente, sem déficit motor, sem alterações no território dos nervos crânicos, perímetro cefálico de $48 \mathrm{~cm}$ e sem sinais de irritação meningea. Nesta ocasiāo, foram realizados os seguintes exames: Eletrencefalograma ( $E E G$ G) - Feito em sono, mostrando persistente e moderada depressão de ritmos rápidos em todo o hemisfério esquerdo, mais acentuada na região temporal (Fig. 1). Exame do liquido cefalorraqueano (LCR) -

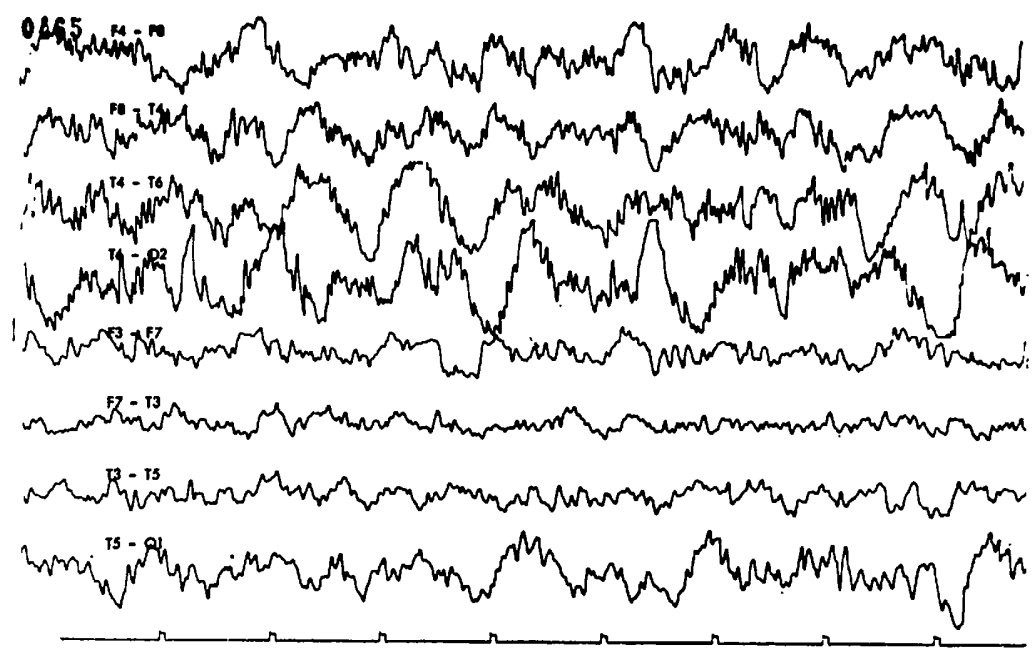

Fig. 1 - Caso O.D.L. Traçado eletrencefalográfico em sono, aos 19 meses de idade, mostrando persistente depressão de ritmos rápidos no hemisfério esquerdo.

Punção sub-occipital; aspecto límpido e incolor; 54 células por $\mathrm{mm}^{3}$ (69\% de linfócitos, $18 \%$ de monócitos, $2 \%$ de neutrófilos e $11 \%$ de eosinófilos); proteinas totais $102 \mathrm{mg} \%$; cloretos e glicose normais; reações de Pandy e Nonne fortemente positivas; reação do benjoim com desvio para a esquerda; reação de Takata-Ara positiva, tipo floculante; reação de 'Weinberg fortemente positiva com $0,1 \mathrm{ml}$; reações de Wassermann, Steinfeld e VDRL, negativas. Reação de Weinberg no soro sangüineo positiva (técnica de Kolmer). Radiografia do crânio normal. Exame protoparasitológico negativo. A paciente foi medicada c $m$ fenobarbital, primidona, dexametasona e sulfadiazina.

Cerca de três meses após a convulsão inicial, surgiu dificuldade progressiva na marcha, tendo o exame neurológico revelado déficit de força no membro inferior direito e reflexo de preensão persecutória no membro superior direito, sem alterações na motilidade deste segmento. Nesta ocasião foi repetido exame de LCR que revelou o mesmo quadro mostrado pelo anterior. Foi feito também novo EEG que revelou, além da depressão anteriormente existente, sinais de atividade irritativa discreta na região temporal esquerda.

Seis meses após a primeira, ocorreu nova crise convulsiva. A partir desta, a paciente, agora com a idade de 2 anos, deixou de compreendr ordens simples, deixou de falar e os reflexos profundos do inembro inferior direito tornaram-se exaltados. O EEG nesta ocasião mostrou atividade irritativa bem evidente, de projeção ora num ou noutro hemisfério, ora bilateral (Fig. 2). Nos meses seguintes, o quadro eletrencefalográfico foi gradativamente intensificado. 


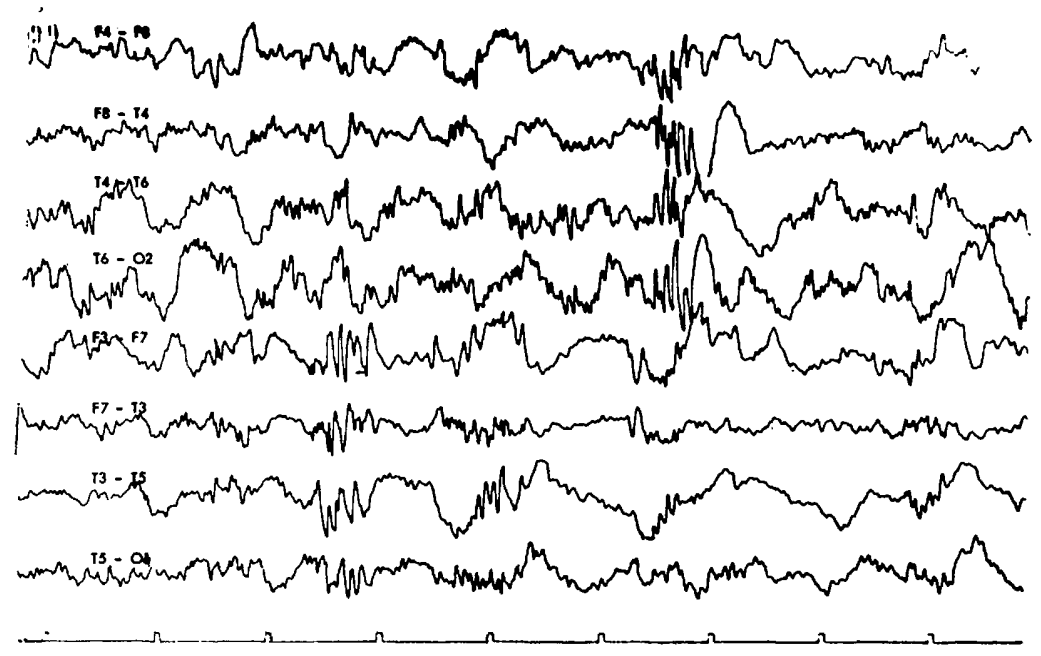

Fig. 2 - Caso O.D.L. Traçado eletrencefalográfico em sono, aos 2 anos e 3 meses, mostrando atividade irritativa de projecão num $e$ noutro hemisfério, além de discreta depressão de ritmos rápidos $\grave{a}$ esquerda.

Um ano e seis meses após o início da doença, com 3 anos de idade, surgiram episódios de flexão da cabeça, em grande número diário, logo após acrescidos de adução dos membros superiores e flexão do tronco, caracterizando típicos espasmos em flexão. Nesta oportunidade o EEG já se mostrava com aspecto característico de hipsarritmia, com pontas e ondas agudas quase continuas, difusas e multifocais, com freqüentes depressōes paroxisticas (Figs. 3 e 4 ).

Meio ano mais tarde, estando a paciente com 3 anos e 6 meses, concomitantemente com as crises já existentes, começaram a ocorrer crises acinéticas (astáticas) em freqüência cada vez maior e, pouco depois, ausências com mioclonias. Nesta ocasião, houve piora das funções psicomotoras, alheamento ao ambiente, agitação, insônia e impossibilidade de manter-e em pé. A paciente não podia falar e só andava quando apoiada; marcha com base alargada, atáxica e ceifante à direita; hipertonia e hiperreflexia no membro inferior direito. O EEG mostrou descargas de polipontas difusas, depressão paroxística e, pela primeira vez, o aparecimento de complexos ponta-onda lenta, de 2 , de $1,5 \mathrm{e}$ até de $1 \mathrm{c} / \mathrm{s}$, bilaterais, predominando em áreas anteriores, geralmente isolados (Fig. 5).

Alguns meses após, surgiram crises convulsivas generalizadas, versivas para a direita e também espasmos em extensão, tornando-se muito raras as crises acinéticas. O EEG neste período mostrou complexos ponta-onda lenta de projeção bilateral e predominantemente anterior, agora ritmados (Fig. 6).

Apesar dos vários esquemas terapêuticos tentados, com sulfas. ACTH, toda a série dos anticonvulsivantes e diazepinicos, nunca houve remissão das crises, estando o quadro clínico inalterado na ocasião em que este trabalho estava sendo escrlto. Durante todo o periodo de acompanhamento, não se observaram sinais clínicos, radiológicos ou fundoscópicos de hipertensão intracraniana, de irritação meningorađicular, déficit visual ou calcificações intracranianas; o perimetro craniano aumen- 
tou de 48 para $50 \mathrm{~cm}$, entre o exame inicial e o de 4 anos após, com 5 anos e 8 meses.

No decorrer da evolução, foram realizados 8 exames de LCR, todos mostrando o mesmo quadro, apenas com variações numéricas.
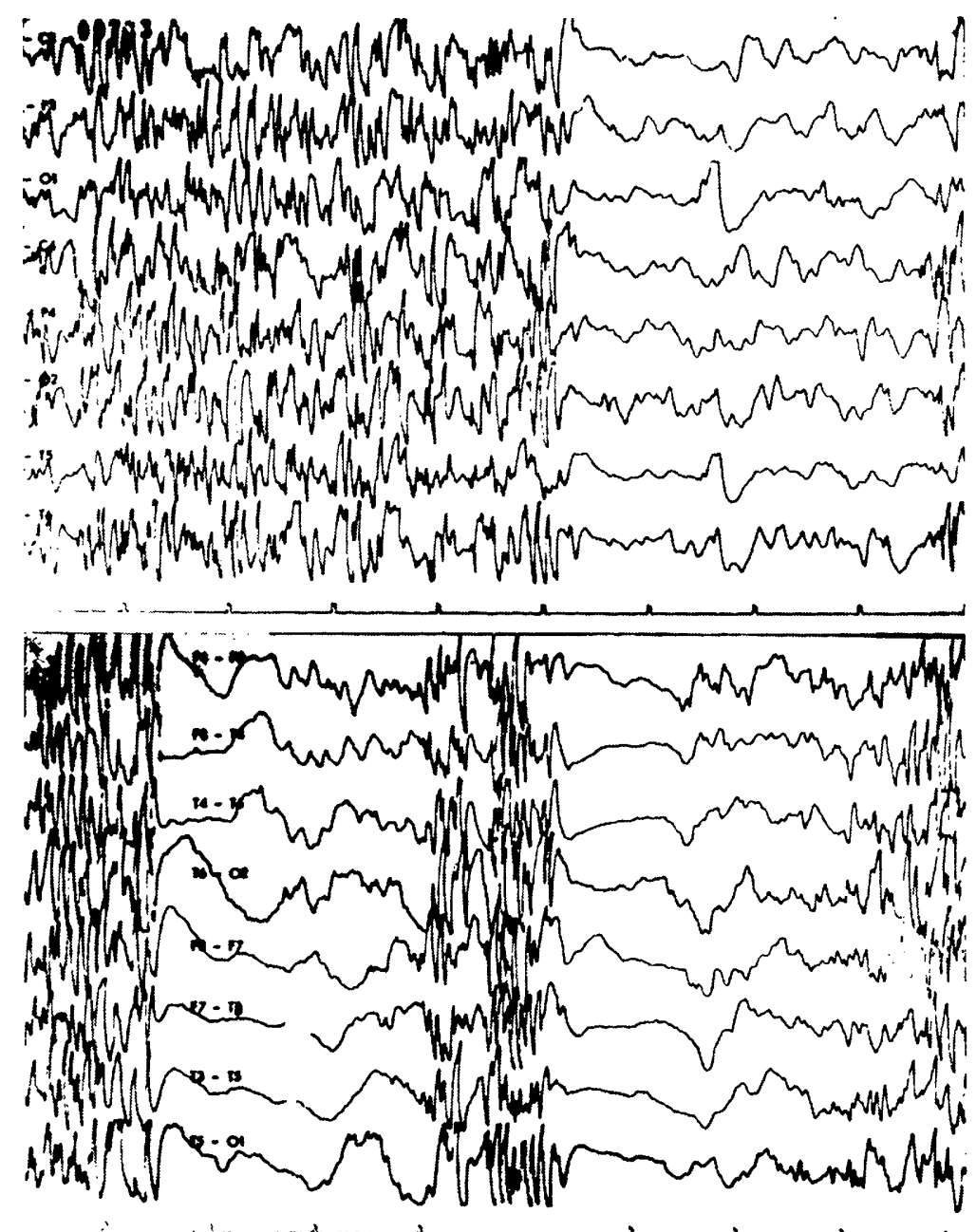

Figs. 3 e 4 - Caso O.D.L. Tracados eletrencefalográfico em sono, aos $s$ anos de idade, mostrando a hipsarritmia fundamental, com surtos de depressão paroxistica difusa, interrompida por periodos livres de descargas (próprio do tracado hipsarritmico em sono), nos quais se nota a relativa pobreza de ritmos no hemisfério esquerdo. 
4pising

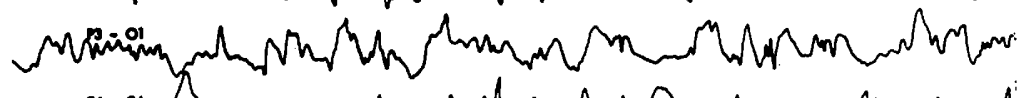

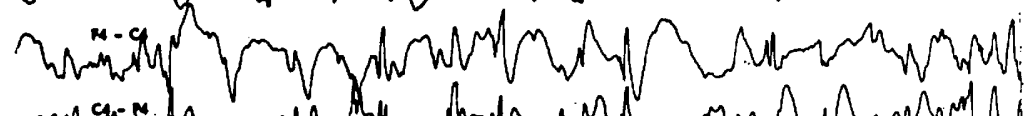

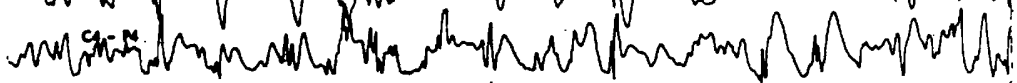

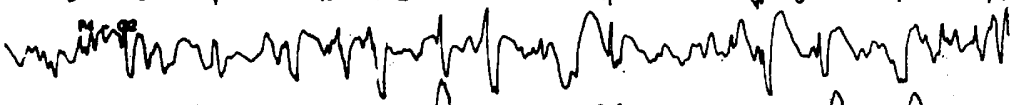

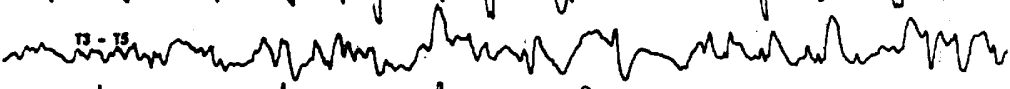

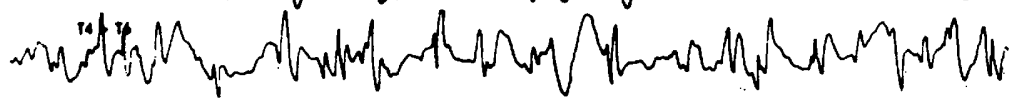

Fig. 5 - Caso O.D.L. Tracado eletrencefalográfico em sono, aos 3 anos e 8 meses de idade, mostrando surtos de complexos ponta-onda lenta a $1 \mathrm{c} / \mathrm{s}$, de projeção bilateral e síncrona, predominando em áreas anteriores, geralmente isolados.

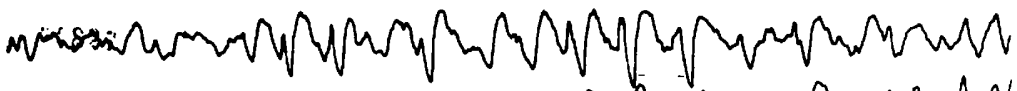

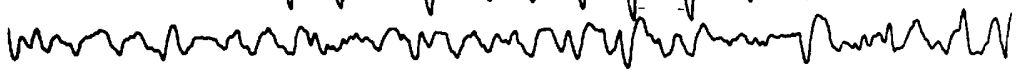

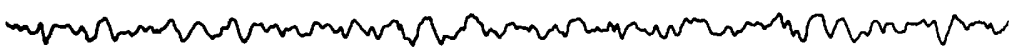

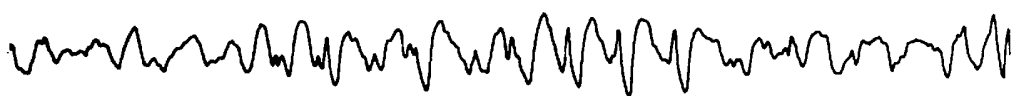

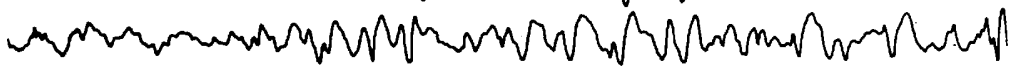

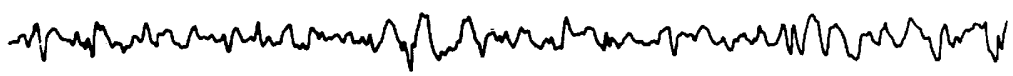

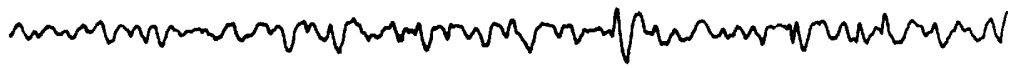

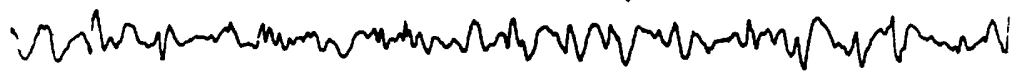

Fig. 6 - Caso O.D.L. Traçado eletrencefalográfico em vigilia, aos 4 anos $e 6$ meses, mostrando complexos ponta-onda lenta ritmados, de projeçâo predominante anterior, sobre um ritmo de base difusamente irregular. 
A evolução de uma síndrome de West para uma de Lennox-Gastaut é fato já bem conhecido e longe de ser raro. Oller-Daurella ${ }^{11}$, em sua notável monografia, encontrou $6 \%$ de pacientes nos quais foi assinalada esta evolução.

Os conhecimentos sobre estas duas sindromes mostram: a) que suas etiologias possíveis são muito variadas quanto à natureza; $b$ ) que muitas das etiologias descritas são condições patológicas que não determinam alteraçōes encefálicas específicas (traumas obstétricos, anóxia, traumatismos cranianos, distrofia alimentar); c) que, em sua maioria, são as mesmas as etiologias descritas em ambas sindromes; $d$ ) que uma síndrome de West pode se transformar numa sindrome de Lennox-Gastaut, enquanto o inverso jamais foi registrado; e) que enquanto a síndrome de West atinge grupos etários mais novos, a síndrome de Lennox-Gastaut acomete geralmente crianças com mais idade.

Estes dados conduzem à idéia de que deve existir um processo patológico básico, até certo ponto independente da etiologia, subjacente a ambas sindromes. De fato, com relação à síndrome de West, Gastaut e col. ${ }^{4}$ levantaram argumentos que levam a considerar o quadro como uma reação imunológica do cérebro imaturo a vários estímulos antigênicos, com uma patogenia semelhante à da encefalomielite alérgica. $O$ mesmo se poderia dizer com relação à sindrome de Lennox-Gastaut.

O principal interesse na apresentação deste caso, no entanto, reside no fato de, apesar das várias infecções e infestações descritas como etiologia, nāo havermos tido conhecimento de nenhuma referência à cisticercose como causa quer da síndrome de West, quer da de Lennox-Gastaut.

\section{R E S U M O}

Os autores relatam a evolução clínico-laboratorial do caso de uma criança que, aos 18 meses de idade, apresentou uma síndrome de West de instalação gradativa, evoluindo ulteriormente para uma síndrome de Lennox-Gastaut, causadas por cisticercose cerebral, etiologia ainda não registrada em nenhum dos quadros.

\section{S U M M A R Y}

West's syndrome changing to Lennox-Gastaut's syndrome in a patient with cerebral cysticercosis

The case of a 6 year old girl with Lennox-Gastaut's syndrome caused by cysticercosis of the central nervous system (CNS) is reported. The illness began at 18 months of age with right sided convulsions during an upper respiratory tract infection, Electroencephalogram (EEG) showed depression of rapid rythm on the left side. There was progressive mental deterioration, right spastic hemiparesis and aphasia. Then the EEG showed progressively 
worsening of generalized irritative activity, with spikes and sharp waves. At the age of 3 years there appeared flexion myoclonic spasms and EEG showed a hypsarythmic pattern. Both clinical and EEG picture allowed the diagnosis of West's syndrome. In the following months the patient presented akinetic seizures, myoclonic jerks and generalized convulsive seizures with turning of the head to the right. EEG revealed slow spike-waves thus leading, at this time, to the diagnosis of Lennox-Gastaut's sindrome. Brain cysticercosis was diagnosed on the basis of 8 repeated positive cerebrospinal fluid examinations.

REFER N CIAS

1. BAIRD, H. W. \& BOROWSKI, L. G. - Infantile myoclonic seizures. J. Pediat. 50:332, 1957.

2. CHRISTIAN, W. - Atlas de Electroencefalografía Clínica. Versão espanhola. Editorial Paz Montalvo, Madrid, 1971. pág. 109.

3. COLEMAN, M. - Infantile spasms associated with 5-hydroxytryptophan administration in patients with Down's syndrome. Neurology (Mineapolis) 21:911, 1971.

4. Gastaut, H.; SAlTiel, J.; RAYBaUd, C.; PITOT, M. \& MAYNADIER, A. A propos du traitement par ACTH des encephalities myocloniques de la première enfance avec dysritmie majeure. Pediatrie 14:35, 1959.

5. GASTAUT, H.; ROGER, J. \& SOULAYROL, R. - Encéphalopathie myoclonique infantile avec hypsarythmie (syndrome de West). In Encyclopedie Médico-Chirurgicale, Neurologie, vol. 1, tome 2, Paris, 1965.

6. GASTAUT, H.; ROGER, J.; SOULAYROL, R.; TASSINARI, C. A.; REGIS, H.; DRAVET, C.; BERNARD, R.; PINSARD, N. \& SAINT-JEAN, M. - Childhood epileptic encephalopathies with diffuse slow spike-waves (otherwise known as "petit mal variant") or Lennox syndrome. Epilepsia (Amsterdam) 7:139, 1966.

7. LENNOX, W. G. \& LENNOX, M. A. - Epilepsy and Related Disorders. Little Brown and Co., Boston, 1960, pág. 165.

8. LOW, N. L. - Infantile spasms. Pediat. Clin. North America 7:373, 1960.

9. MILLICHAP, J. G.; BICKFORD, R. G.; KLASS, D. W. \& BOCKUS, R. E. Infantile spasms, hypsarhythmia and mental retardation. A study of etiologic factors in 61 patients. Epilepsia (Amsterdam) 3:188, 1962.

10. NIEDERMEYER, E. - The Generalized Epilepsies. Charles C. Thomas Publisher, Springfield (Illinois), 1972. pág. 58.

11. Oller-DAURElla, L. - El Sindrome de Lennox y sus Diferentes Formas Clínico-eletroencefalograficas. Editorial Espaxs, Barcelona, 1967. pág. 33.

12. SOREL, L. - L'épilepsie myokinetique grave de la première enfance avec pointe-onde lente et son traitement (petit mal variant). Rev. neurol. (Paris), 110:215, 1964 .

Clínica Neurológica - Hospital do Servidor Público Estadual - Rua Pedro de Toledo 1800 - 04039 São Paulo, SP - Brasil. 